\title{
Arabidopsis bZIP11 Is a Susceptibility Factor During Pseudomonas syringae Infection
}

\author{
Matthew J. Prior, ${ }^{1,2,3}$ Jebasingh Selvanayagam, ${ }^{2,4}$ Jung-Gun Kim, ${ }^{3}$ Monika Tomar, ${ }^{4}$ Martin Jonikas, ${ }^{2,5}$ \\ Mary Beth Mudgett, ${ }^{3}$ Sjef Smeekens, ${ }^{4,+}$ Johannes Hanson, ${ }^{4,6,+}$ and Wolf B. Frommer ${ }^{2,3,7,+}$ \\ ${ }^{1}$ Department of Botany and Plant Sciences, University of California Riverside, Riverside, CA 92507, U.S.A. \\ ${ }^{2}$ Department of Plant Biology, Carnegie Institution for Science, Stanford, CA 94305, U.S.A. \\ ${ }^{3}$ Department of Biology, Stanford University, Stanford, CA 94305, U.S.A. \\ ${ }^{4}$ Molecular Plant Physiology, Department of Biology, Utrecht University, Utrecht, The Netherlands \\ ${ }^{5}$ Department of Molecular Biology, Princeton University, 119 Lewis Thomas Laboratory, Washington Road, Princeton, NJ, \\ U.S.A. \\ ${ }^{6}$ Umeå Plant Science Centre, Department of Plant Physiology, Umeå University, Umeå, Sweden \\ ${ }^{7}$ Molecular Physiology, Heinrich Heine Universität, 40225 Düsseldorf, Germany
}

Accepted 4 January 2021.

\begin{abstract}
The induction of plant nutrient secretion systems is critical for successful pathogen infection. Some bacterial pathogens (e.g., Xanthomonas spp.) use transcription activator-like (TAL) effectors to induce transcription of SWEET sucrose efflux transporters. Pseudomonas syringae pv. tomato strain DC3000 lacks TAL effectors yet is able to induce multiple SWEETs in Arabidopsis thaliana by unknown mechanisms. Because bacteria require other nutrients in addition to sugars for efficient reproduction, we hypothesized that Pseudomonas spp. may depend on host transcription factors involved in secretory programs to increase access to essential nutrients. Bioinformatic analyses identified the Arabidopsis basic-leucine zipper transcription factor bZIP11 as a potential regulator of nutrient transporters, including SWEETs and UmamiT amino acid transporters. Inducible downregulation of bZIP11 expression in Arabidopsis resulted in reduced growth of $P$. syringae pv. tomato strain DC3000, whereas inducible overexpression of $b Z I P 11$ resulted in increased bacterial growth, supporting the hypothesis that bZIP11-regulated transcription programs are essential for maximal pathogen titer in leaves. Our data are consistent with a model in which a
\end{abstract}

\section{J. Prior and J. Selvanayagam contributed equally to this work.}

${ }^{\dagger}$ Corresponding authors: S. Smeekens; j.c.m.smeekens@uu.nl; J. Hanson; johannes.hanson@umu.se; and W. B. Frommer; frommew@hhu.de

Funding: This work was supported by the National Science Foundation research grants IOS-1258103 to W. B. Frommer, IOS-1555957 to M. B. Mudgett, and IOS-1810468 to M. J. Prior; the Office of Basic Energy Sciences of the U.S. Department of Energy grant number DE-FG0204ER15542; Deutsche Forschungsgemeinschaft (German Research Foundation) under Germany's Excellence Strategy-EXC-2048/1 (project ID 390686111); Alexander von Humboldt-Stiftung via the Alexander von Humboldt Professorship to W. B. Frommer; a Stanford Graduate Fellowship to M. J. Prior; and the Nederlandse Organisatie voor Wetenschappelijk Onderzoek (Netherlands Organization for Scientific Research) (grant number 854.10.011) to S. Smeekens.

*The $\boldsymbol{e}$-Xtra logo stands for "electronic extra" and indicates there are supplementary material published online.

The author(s) declare no conflict of interest.

(c) (1) $\Theta$ Copyright $(92021$ The Author(s). This is an open access article distributed under the CC BY-NC-ND 4.0 International license. pathogen alters host transcription factor expression upstream of secretory transcription networks to promote nutrient efflux from host cells.

Keywords: bacterial pathogenesis, bZIP transcription factor, plant nutrient secretion systems, plant responses to pathogens, secretion and cell wall changes, susceptibility factor, type 3 secretion

Plant pathogens face two distinct challenges with respect to successful reproduction during invasion of their hosts. First, they must evade or suppress the host immune response to survive. Second, they must rapidly acquire sufficient nutrients to fuel their growth, often to very high titers in the host tissue. Substantial efforts have provided a detailed picture of how plant pathogens overcome immune responses (Dou and Zhou 2012; Jones and Dangl 2006); however, we are only beginning to understand the strategies pathogens employ to secure access to nutrients from their hosts (Bezrutcyk et al. 2018).

One strategy used by bacterial pathogens of the genus Xanthomonas to access plant nutrients is the deployment of transcription activator-like (TAL) effectors. TAL effectors induce the transcription of SWEET sucrose transporters in diverse hosts (e.g., rice, cassava, cotton, and pepper), where each TAL effector induces a specific host sugar transporter gene (Cernadas et al. 2014; Chen et al. 2010; Cohn et al. 2014; Cox et al. 2017). TALinduced expression of rice OSSWEET genes is required for Xanthomonas oryzae pv. oryzae pathogenesis, indicating that SWEETs are key host susceptibility factors during Xanthomonasrice interactions (Cernadas et al. 2014; Chen et al. 2010). Rice lines carrying mutations in the TAL effector-binding site in the promoters of OsSWEET11, OsSWEET13, or OsSWEET14 are resistant to X. oryzae pv. oryzae (Chen et al. 2010; Cernadas et al. 2014). Promoter variants in SWEET genes yield broad-spectrum resistance against bacterial leaf blight (Eom et al. 2019; Oliva et al. 2019). SWEET genes are also induced in other plants in response to diverse pathogens, including bacteria, protists, and fungi (Chen et al. 2010; Chong et al. 2014; Cox et al. 2017; Siemens et al. 2006). The genomes of these pathogens apparently lack TAL effector homologs (Buell et al. 2003; Hahn et al. 2014; Schwelm et al. 2015), implicating alternate mechanisms for the induction of SWEET genes. 
Given that pathogens require access to essential micro- and macroelements in addition to sugars for growth, we hypothesized that pathogens may target transcription factors that alter nutrient availability at the local site of infection. To test this hypothesis, we asked whether the apoplasmic pathogen $\mathrm{Pseu}$ domonas syringae pv. tomato strain DC3000 induces multiple nutrient transporters, including SWEETs, in an effectordependent manner (i.e., requiring the use of its type-three secretion system [T3SS]). We used a bioinformatic approach, reasoning that $P$. syringae pv. tomato strain DC3000 might upregulate the expression of multiple SWEET genes or other nutrient transporters by targeting a transcription factor that normally functions upstream of metabolic programs found in secretory cell types such as seed coat or tapetum. We predicted that ectopic induction of such a transcription factor in local leaf cells could effectively increase the metabolic pools of nutrients in the reprogrammed cells and simultaneously induce transporters to efflux nutrients into the apoplasm for extracellular pathogens such as $P$. syringae pv. tomato strain DC3000 (Fig. 1A).

Arabidopsis thaliana bZIP11, a basic leucine-zipper (bZIP) domain transcription factor, is known to be a crucial transcriptional regulator of genes involved in plant sugar and amino acid metabolism (Hanson et al. 2008; Ma et al. 2011). Here, we report that bZIP11 acts as a susceptibility factor during P. syringae pv. tomato strain DC3000 infection. We show that, during development, bZIP11 influences the transcription of multiple nutrient transporters with hallmarks of nutrient efflux from secretory cells. We also show that bZIP11 expression is required for maximal $P$. syringae pv. tomato strain DC3000 growth in Arabidopsis leaves and that overexpression of bZIP11 leads to increased bacterial growth. Our data suggest that bZIP11-regulated transcription programs play an important role in bacterial pathogenesis.

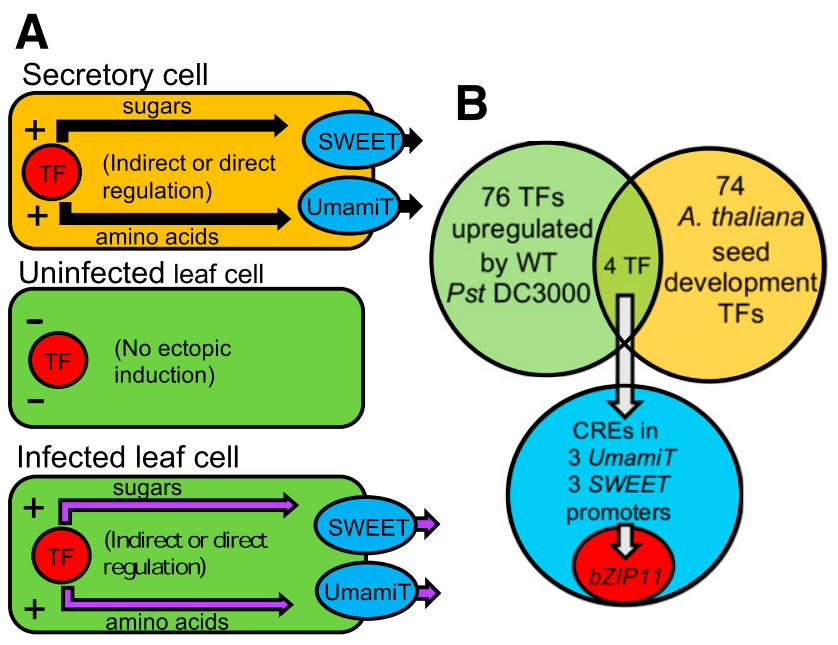

Fig. 1. Screen for a transcription factor (TF) that may influence secretory cell functions and is induced by the bacterial pathogen Pseudomonas syringae pv. tomato strain DC3000. A, Diagram showing basic movement of nutrients from secretory cells during seed development (top), and those proposed in uninfected leaf cells (middle) and infected leaf cells (bottom) by a TF that influences downstream SWEET and UmamiT transcription. B, Bioinformatic analysis of microarray data compared 74 TFs previously identified in distinct groups by hierarchical clustering during Arabidopsis seed development against 76 TFs induced by wild-type (WT) strain DC3000 in a type-three secretion system-dependent manner in Arabidopsis leaves. One of the four TFs found in both datasets, $b Z I P 11$, was identified as a single best candidate TF.

\section{RESULTS}

Bioinformatic analyses identified bZIP11 mRNA induction during infection.

Three criteria were used to bioinformatically identify transcription factors in Arabidopsis that are selectively induced by pathogens and which, in turn, could ectopically induce transcription of transporter genes for nutrient efflux, either directly or indirectly (Fig. 1A). First, we examined a set of transcription factors expressed in distinct hierarchical clusters during Arabidopsis seed development, because the seed coat is a secretory cell layer that expresses specific nutrient exporters, as seen in the study by Le et al. (2010, their table S9) and, in particular, SWEET sucrose and UmamiT amino acid transporters (Besnard et al. 2018; Chen et al. 2015; Müller et al. 2015). We then compared this group to another set of transcription factors reported to be induced in Arabidopsis leaves during $P$. syringae pv. tomato strain DC3000 in a T3SS-dependent manner, as found in the study by Truman et al. (2006, their table 3). The overlap between these two sets identified four candidate transcription factors: bZIP11, bZIP9, AGL18, and At3g19910 (Fig. 1B; Supplementary Table S1). We identified transcription factor binding sites (i.e., cis regulatory elements) enriched in promoters of putative nutrient transporters, including three SWEETs and three UmamiTs, and found an enrichment of bZIP sites (Supplementary Table S2) (Zambelli et al. 2009). Of these six transporters, SWEET15, UmamiT18, and UmamiT29 have known feeding roles during seed development, raising the possibility that they might also play a role during pathogen infection (Chen et al. 2015; Ladwig et al. 2012; Müller et al. 2015).

Among the candidates identified, we selected bZIP11 as the top candidate for further studies because it fulfilled all of our bioinformatic and physiological criteria: (i) expressed in a secretory tissue (such as seed coat during seed filling), (ii) induced by the pathogen, and (iii) the candidate gene list is enriched for bZIP binding sites in their promoters. We also prioritized bZIP11 because its expression is regulated translationally by sucrose levels and is known to be crucial for controlling the carbon and nitrogen balance and regulating amino acid metabolism (Hanson et al. 2008; Ma et al. 2011). Prior work showed that overexpression of bZIP11 in Arabidopsis resulted in a marked increase in metabolic pools toward substrates for glycolysis, including increased glucose, fructose, and sucrose content, while multiple amino acids concentrations are also increased (Ma et al. 2011). Moreover, overexpression of a tobacco bZIP11 homolog resulted in increased leaf sugar levels (Thalor et al. 2012). Taken together, the phenotypes observed in plants with bZIP up- and downregulation are consistent with functional characteristics of a transcription factor that alters metabolite levels and transporter gene expression.

\section{bZIP11 is induced during $P$. syringae pv. tomato strain DC3000 infection in an effector-dependent manner.}

An analysis of published microarray datasets indicated that, in three of four studies, bZIP11 was induced by P. syringae pv. tomato strain DC3000 in a T3SS effector-dependent manner (Cumbie et al. 2011; Huang et al. 1992; Kemmerling et al. 2007; Thilmony et al. 2006; Truman et al. 2006). To independently validate these findings, real-time PCR analyses were performed using Arabidopsis Col-0 leaves infected with wild-type $P$. syringae pv. tomato strain DC3000 or P. syringae pv. tomato strain DC3000 $\Delta h r c U$, which is defective in typethree secretion (Huang et al. 1992). In three separate biological repeats, bZIP11 mRNA levels were significantly increased in 
leaves following inoculation with wild-type $P$. syringae pv. tomato strain DC3000 but not the $P$. syringae pv. tomato strain DC3000 $\Delta h r c U$ mutant, indicating T3SS dependence (Supplementary Fig. S1). Taken together, we hypothesize that $P$. syringae pv. tomato strain DC3000 uses T3SS effector proteins to induce $b Z I P 11$ transcription.
bZIP11 is necessary for normal development, metabolism, and transporter gene expression.

To test the importance of bZIP11 in pathogen susceptibility, estradiol-inducible artificial microRNA (amiRNA) Arabidopsis lines targeting bZIPII were generated, because homozygous null mutants could not be recovered. Two independent transgenic lines
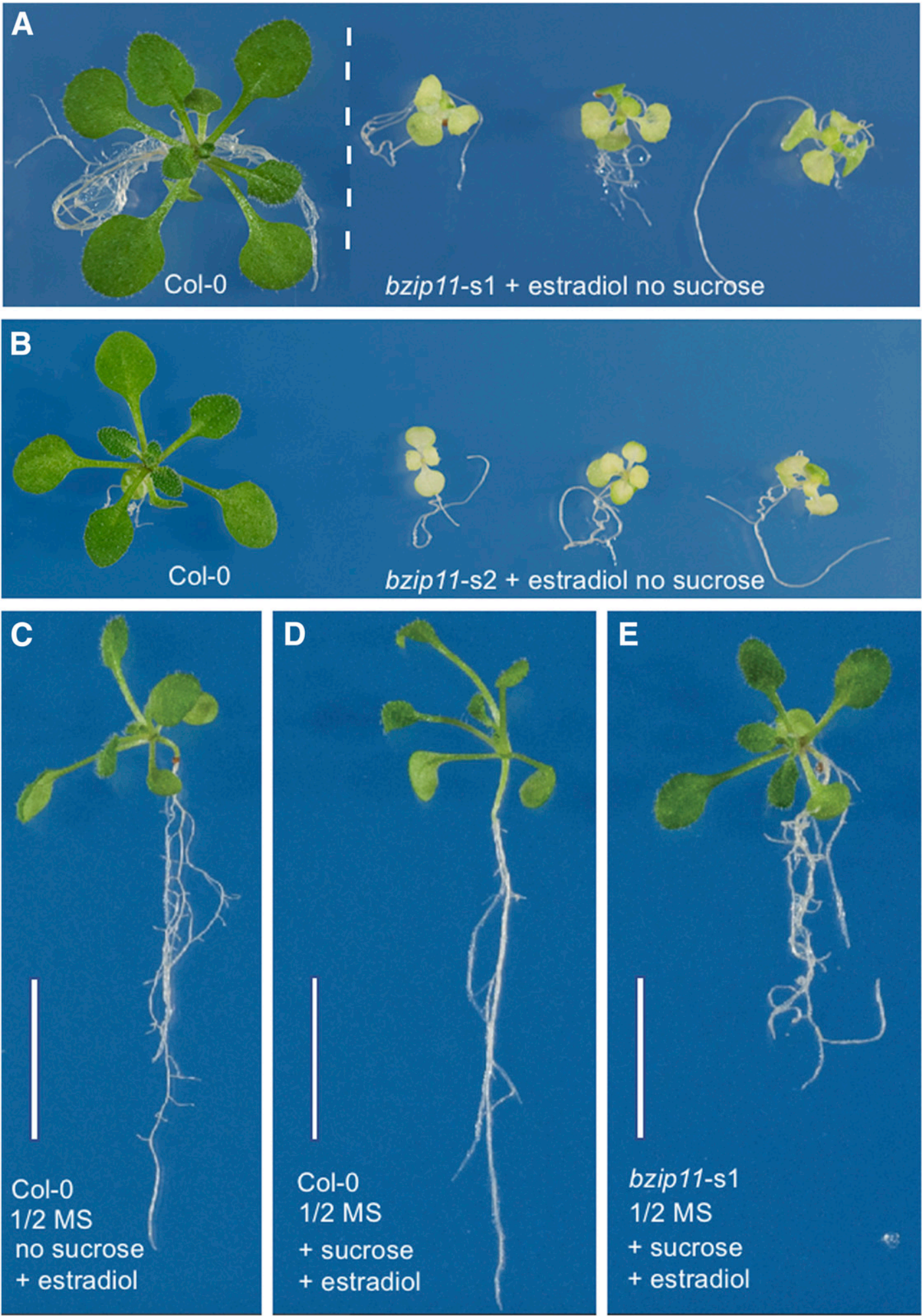

Fig. 2. bZIP11 artificial microRNA-inducible expression lines in the presence or absence of sucrose and estradiol. A and B, Twenty-one-day-old wild-type (Col-0), bzip11-s1, and bzip11-s2 representative seedlings grown on media supplemented with $50 \mu \mathrm{M}$ estradiol. C, Wild-type grown on media supplemented with $50 \mu \mathrm{M}$ estradiol. D, Wild-type grown on media supplemented with $50 \mu \mathrm{M}$ estradiol and $30 \mathrm{mM}$ sucrose. E, Transgenic bzip11-s1 grown on media supplemented with $50 \mu \mathrm{M}$ estradiol and $30 \mathrm{mM}$ sucrose. Scale bar $=1 \mathrm{~cm}$ and $1 / 2 \mathrm{MS}=$ half-strength Murashige-Skoog medium. 
(bzip11-s1 and bzip11-s2) were chosen after analyzing bZIP11 mRNA abundance by real-time PCR (Supplementary Fig. S2A). With the addition of $50 \mu \mathrm{M}$ estradiol, mRNA abundance in bzip11-s1 and bzip11-s2 was approximately 69 and $42 \%$ of wildtype levels, respectively (Supplementary Fig. S2A). Both amiRNA lines were smaller in stature compared with the wild-type Col-0 background at 4 weeks of growth in soil, consistent with expression of the amiRNA without the hormone (Supplementary Fig S2B). This could be due to a positional effect of insertion of the transgene into the chromosome or could be due to a low level of constitutive repression of bZIP11 in absence of estradiol (Supplementary Fig. S2B). When germinated on $50 \mu \mathrm{M}$ estradiolcontaining media, both bzip11-s1 and bzip11-s2 seedlings showed chlorotic leaves and compromised root and shoot growth compared with wild-type Col-0 (Fig. 2A and B). Addition of sucrose to media containing $50 \mu \mathrm{M}$ estradiol rescued the growth defects of the bzip11-s1 amiRNA line (Fig. 2E). These results intimate that bZIP11 is essential for normal growth of Arabidopsis and its effects can be bypassed through the addition of carbohydrates. They also are consistent with a role of bZIP11 upstream of the sucrose synthesis or export during growth regulation in Arabidopsis.

To identify genes regulated by bZIP11, mRNAs differentially regulated in Col-0 relative to the bZIP11 mutants were compared (microarray data for bzip11-s1 and RNA sequencing [RNAseq] data for bzip11-s2). An unbiased gene ontology (GO) analysis was performed (Supplementary File S1). GO analysis of altered gene expression in bzip11-s1 indicates that several categories of genes connected to enzyme activity and transport such as oxidoreductase, peroxidase, and transmembrane transport are enriched. Other terms that are highly enriched in the dataset are heme and tetrapyrrole binding, responses to various stresses (chemical and hypoxia) (Supplementary File S1).

\section{Validation of sequencing experiments.}

A subset of the genes altered in sequencing experiments was validated by real-time PCR, to confirm the downregulation patterns for bzip11-s1 (Supplementary Fig. S3). We cannot exclude that immunity may also be playing a role in the mutants because knockdown of bZIP11 gene expression also alters a significant amount of immunity-related transcripts (Supplementary File S1). At the same time, the abundance of mRNAs for several transporters, including five UmamiTs, was reduced in the microarray analysis of bzipl1-sl in the presence of $50 \mu \mathrm{M}$ estradiol (Table 1). UmamiT14, UmamiT18, and UmamiT29 play critical roles in supplying amino acids to the developing seed (Ladwig et al. 2012; Müller et al. 2015). The abundance of both UmamiT18 and UmamiT29 mRNAs were increased in wild-type Arabidopsis leaves during P. syringae pv. tomato strain DC3000 induction (Kemmerling et al. 2007). The abundance of mRNAs for representatives from two types of

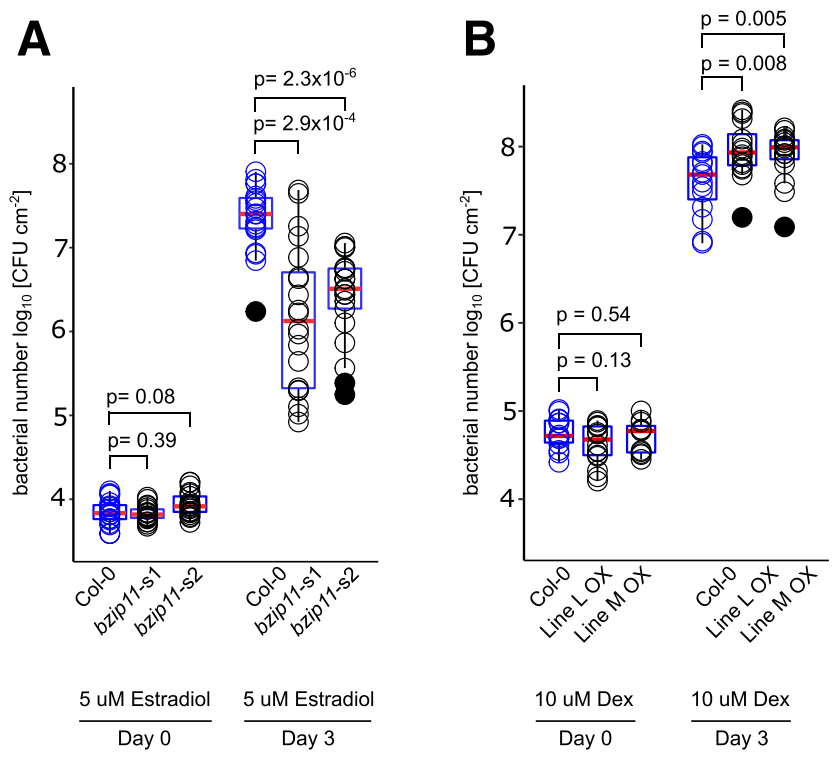

Fig. 3. A, Box plot showing levels of Pseudomonas syringae pv. tomato strain DC3000 in the leaves of Arabidopsis wild-type Col-0- or bZIP11inducible artificial microRNA (amiRNA) lines bzip11-s1 and bzip11-s2. Bacterial titer at day 0 (start of infection) and day 3. Bacterial suspensions contained $5 \mu \mathrm{M}$ estradiol. Data represent $\log _{10} \mathrm{CFU} / \mathrm{cm}^{2}$ from 61 individual plants. Experiments were repeated in four independent biological repeats. Blue boxes represent the 25 th percentile (top) and 75 percentile (bottom) of the sample set respectively. The red line in each box is the median sample. Each circle is an independent count of CFU per square centimeter from a single leaf of 1 of the 61 individual plants; wild-type counts are in blue and amiRNA counts are in black. Closed circles represent sample outliers. Statistical significance by a two-way Student's $t$ test is provided. B, Box plot showing levels of $P$. syringae pv. tomato strain DC3000 in the leaves of Arabidopsis wild-type Col-0 or bZIP11 dex-inducible overexpression line $\mathrm{M}$ and line L. Bacterial suspensions contained $10 \mu \mathrm{M}$ dex. Data represent $\log _{10} \mathrm{CFU} / \mathrm{cm}^{2}$ from 47 individual plants; wild-type counts are in blue and overexpression counts are in black. Bacterial titer at day 0 (start of infection) and day 3. Data are from three independent replicates, except the day 0 for line $M$ is only two replicates. Blue boxes represent the 25 th percentile (top) and 75 percentile (bottom) of the sample set. The red line in each box is the median sample. Each circle is an independent count of CFU per square centimeter from a single leaf of 1 of the 47 individual plants. Statistical significance by a two-way Student's $t$ test is provided.

Table 1. Nutrient transporters downregulated in bzip11-s1 knockdown transgenic plants compared with the wild-type plants ${ }^{\mathrm{a}}$

\begin{tabular}{lll}
\hline Gene ID & Annotation & \multicolumn{1}{c}{ Description } \\
\hline AT2G39510 & UMAMIT14 & \multicolumn{1}{c}{ Fold change } \\
AT1G44800 & UMAMIT18 & \multicolumn{2}{c}{ Amino acid transporter } \\
AT4G01440 & UMAMIT31 & Amino acid transporter \\
AT4G30420 & UMAMIT34 & Amino acid transporter \\
AT4G01430 & UMAMIT29 & Amino acid transporter \\
AT1G08090 & NRT2:1 & Amino acid transporter \\
AT1G32450 & NRT1.5 & Nitrate transporter \\
AT1G22550 & Putative & Nitrate transporter \\
AT1G72140 & Putative & Putative peptide/nitrate transporter \\
AT3G24300 & AMT1;3 & Putative peptide/nitrate transporter \\
AT1G64780 & AMT1;2 & Ammonium transporter \\
AT3G62270 & BOR2 & Ammonium transporter \\
AT3G46900 & COPT2 & Putative boron transporter \\
AT3G23430 & PHO1 & Copper transporter \\
AT5G59520 & ZIP2 & Phosphate transporter \\
\hline
\end{tabular}

${ }^{a}$ Fold change is equal to the average signal of the wild type/average signal of bZIP11-s1. A negative sign (-) represents a downregulation of the transporter's expression compared with the wild-type control. 
nitrate/peptide, ammonium, copper, phosphate, and zinc transporters were also reduced in both $b Z I P 11$ amiRNA lines (Table 1). However, under the conditions tested, none of the SWEET genes were significantly downregulated in the amiRNA lines. It is at present unclear why we did not observe significant changes in SWEET gene expression in these experiments. At this point, we cannot rule out the possibility that the time points chosen, or the estradiol dose used for estradiol-triggered repression of bZIP11 expression, did not capture effects on SWEET gene expression. These studies show that UmamiTs and a set of transporters are downregulated in both the bzip11-s1 and bzip11-s2 mutants.

\section{P. syringae pv. tomato strain DC3000 growth is impaired in bZIP11 mutants.}

To determine whether silencing of bZIPI1 would affect susceptibility of Arabidopsis to bacteria, bzip11-s1, bzip11-s2, and Col-0 leaves were infected with a suspension of $P$. syringae pv. tomato strain DC3000 containing $50 \mu \mathrm{M}$ estradiol. At 3 days postinfection, bzip11-s1 and bzip11-s2 leaves contained approximately 10 -fold lower $P$. syringae pv. tomato strain DC3000 titers compared with wild-type leaves (Fig. 3A). Prior to infection, leaves of bzip11-s1 and bzip11-s2 or the wild-type control did not show apparent signs of chlorosis, a phenotype that could indicate stress (Supplementary Fig. S2B). Chlorosis was observed for mutants when grown for 21 days on plates with estradiol in the absence of sucrose (Fig. 2A and B). At 3 days postinfection, all three adult plants showed a similar degree of pathogen-induced leaf collapse and yellowing at the leaf margins (Supplementary Fig. S4), typical effects observed for leaves due to the high inoculum of the pathogen. Notably, only the inducible repression of bZIP11 expression upon infiltration with $P$. syringae pv. tomato strain DC3000 significantly reduced pathogen growth in leaves. Our findings indicate that bZIP11 expression in adult plants is required for maximal growth of $P$. syringae pv. tomato strain DC3000 in leaves. Next, we directly tested immune responses during $P$. syringae pv. tomato strain DC3000 infection in bzip11-s1 and bzip11-s2 by quantifying the growth of $P$. syringae pv. tomato strain DC3000 $\Delta h r c U$, a strain that grows poorly in leaves due to the lack of a functional T3SS (Mudgett and Staskawicz 1999). Titers of $P$. syringae pv. tomato strain DC3000 $\Delta h r c U$ were similar in bzip11-s1, bzip11-s2, and Col-0, indicating that the bzipl1 amiRNA lines did not display enhanced resistance (Supplementary Fig. S5). Reactive oxygen species (ROS) production in leaves also appeared unaffected (Supplementary Fig. S6). Therefore, the reduction in growth of $P$. syringae pv. tomato strain DC3000 in bzip11-s1 and bzip11-s2 compared with that in Col-0 is not likely caused by an enhanced host immune response. Rather, these findings suggest that reduced pathogen growth is due to the loss of bZIP11-dependent transcription programs, which are required to promote pathogen replication.

\section{$P$. syringae pv. tomato strain DC3000 growth is enhanced in bZIP11 overexpression lines.}

Next, we tested whether overexpression of bZIP11 is sufficient to increase the growth of the pathogen. To test this, we used the previously established dex-inducible line $\mathrm{L}$ and line $\mathrm{M}$ (Hanson et al. 2008). We infected Col-0 line $\mathrm{L}$ and line $\mathrm{M}$ with a suspension of $P$. syringae pv. tomato strain DC3000 containing $1 \mu \mathrm{M}$ dex but observed no statistically significant change in pathogen growth across the three genotypes. However, we note that, on average, there were slightly more bacteria in the overexpression lines than in the Col-0 control (Supplementary Fig. S7). The dex concentration was next increased to $10 \mu \mathrm{M}$ and we observed approximately a 240 and $189 \%$ increase in pathogen titer in line $\mathrm{L}$ and line $\mathrm{M}$, respectively, compared with the Col-0 control at 3 days postinfection (Fig. $3 \mathrm{~B}$ ). Line $\mathrm{L}$ had, on average, a $27 \%$ higher titer of bacteria than line M. This finding is consistent with the previous observation that line $\mathrm{L}$ displayed stronger expression of the transgene than line $\mathrm{M}$, although this difference in bacterial titer was not statistically significant (Hanson et al. 2008). The dose-dependent increase in $P$. syringae pv. tomato strain DC3000 growth in the dex-inducible bZIP11 lines compared with the Col-0 control further suggests that overexpression of bZIP11 is sufficient to increase pathogen growth. Thus, we conclude that bZIP11 is a host susceptibility factor that impacts the outcome of bacterial infection in Arabidopsis.

\section{DISCUSSION}

The strategies that allow pathogens to acquire nutrients from the host to sustain invasion and reproduction in host plants are still largely unknown. The understanding of the bacterial genefor-gene regulatory system that allows Xanthomonas spp. to infect a variety of plant hosts is now a basis for engineering resistance in rice and specialized diagnostic toolkits for $X$. oryzae pv. oyzae infection (Chen et al. 2010; Eom et al. 2019; Oliva et al. 2019). Because there are hundreds of economically important plant pathogens and only a few are known to possess TAL-like effectors, the Xanthomonas TAL effectorhost SWEET gene-for-gene susceptibility mechanism is more likely an exception rather than a general rule. Hijacking the transcriptional programs of secretory cell types may represent an alternate mechanism to access a broad spectrum of plant nutrients beyond soluble carbohydrates.

Overexpression of bZIP11 or bZIP11 homologs led to a marked increase in sugars and amino acids in several different plants systems, indicating that they influence downstream nutrient pools (Hanson et al. 2008; Ma et al. 2011; Thalor et al. 2012). The microarray and RNAseq analyses of wild-type and bzip11 mutants revealed that bZIP11 is required for the expression of multiple nutrient transporter genes, as well as genes involved in metabolic processes and growth (Table 1; Supplementary Table S4). Similarly, previous studies reported that overexpression of bZIP11 results in an overall increase in sugars and multiple amino acids relative to wild-type plants (Hanson et al. 2008). Notably, P. syringae pv. tomato strain DC3000 growth was significantly increased in leaves overexpressing bZIP11 (i.e., bZIP11 dex-inducible lines treated with $10 \mu \mathrm{M}$ dex) compared with wild-type Col-0 lines. These data indicate that activation of bZIP11-dependent nutrient pathways increases susceptibility to $P$. syringae pv. tomato strain DC3000. These data are consistent with our model showing that bacterial modulation of host bZIP11 mRNA expression results in an increase in available nutrient pools and the expression of a wide range of transporters.

Interestingly, alteration of host nutrient transporter expression seems to be crucial in several host-pathogen systems. The Arabidopsis hexose $/ \mathrm{H}^{+}$symporter STP13 was found to compete for sugars in the apoplasm during $P$. syringae pv. tomato strain DC3000 infection as a key part of the host's defense response, consistent with the idea that competition over nutrient availability is a crucial aspect of the host-pathogen relationship (Yamada et al. 2016). Additionally, mutations in the wheat STP13 homolog confer broad-spectrum resistance against fungal rusts (Moore et al. 2015). Recently, the unmutated TaSTP13 gene in wheat was shown to confer susceptibility to stripe rust, potentially by increasing apoplasmic hexose availability (Huai et al. 2020). In rice, OsSWEET13 and OsSWEET15 were reported to be directly regulated by OsbZIP73 and induced during salt and drought stress (Mathan et al. in press). In our sequencing experiments however, none of the 
SWEET genes were significantly downregulated in the two amiRNA lines. Interestingly, a previous study reported that the ectopic overexpression of bZIP11 in line M and line L seedlings resulted in the downregulation of SWEET1 and SWEET12 mRNA levels when the seedlings were treated with $10 \mu \mathrm{M}$ dex induction for $2 \mathrm{~h}$ (Hanson et al. 2008). Additional work may help distinguish the differences between rice and Arabidopsis with regards to bZIP and SWEET regulation. The modulation of host nutrient transporters and the accompanying changes in metabolite availability have also been proposed to play important roles in the host immune response and impact growth and development (Herbers et al. 1996; Kocal et al. 2008; Liu et al. 2016; Sonnewald et al. 2012). Interestingly, a pepper bZIP protein, CabZIP2, is required for full resistance in pepper against $X$. euvesicatoria, and transgenic overexpression of CabZIP2 in Arabidopsis increased resistance to P. syringae pv. tomato strain DC3000 (Lim et al. 2015). Thus, it is possible that bZIP proteins may play roles in either resistance or susceptibility in different pathosystems.

We note that knockdown of bZIP11 gene expression also results in the significant accumulation of immunity-related transcripts, (Supplementary File S1); however, this did not appear to cause an enhanced resistance response in the bZIP11 amiRNA knock-down mutants. For example, flg22-elicited ROS production was similar in the mutants compared with in wild-type plants (Supplementary Fig. S6), indicating no changes in pathogen-associated molecular pattern-triggered immunity. Moreover, the titers of $P$. syringae pv. tomato strain DC3000 $\Delta h r c U$ were similar in bzip11-s1, bzip11-s2, and Col0 , indicating that the bzip11 amiRNA lines did not display any significant changes in defenses to this strain (Supplementary Fig. S5). Nevertheless, at this point, we cannot exclude the possibility that immunity may also play a role in these mutants. An alternative scenario could be that bZIP11 may function as a repressor of some immunity genes or may be guarded by a nucleotide-binding leucine-rich repeat receptor. Therefore, it is possible that bZIP11 may play separate roles as both a repressor of immunity and an activator of nutrient pathways for the pathogen.

Previous work defines genes that have been shown to have a negative regulatory role in the immune response as a class of susceptibility genes (van Schie and Takken 2014). Several studies provided examples of susceptibility $(S)$ genes such as E3 ubiquitin ligases and mitogen-activated protein kinase phosphatases (Anderson et al. 2011; Bartels et al. 2009; Trujillo et al. 2008; Zeng et al. 2004). Therefore, if bZIP11 were to play a repressive role in the immune response, it would reasonably fit into this category of $S$ genes and may be considered a susceptibility factor. Alternatively, if bZIP11 regulates nutrient secretion programs, it could also be considered an $S$ gene, similar to the clade three SWEETs (Bezrutczyk et al. 2018).

Testing a direct link between pathogen growth and the host's nutritional status remains challenging because we predict that pathogen induction of bZIPII expression alters not only nutrient transporter expression but also the metabolic state of the infected cells. Thus, overexpression of candidate nutrient transporters alone is not likely to be sufficient for rescuing the bzip11 mutant phenotype in pathogen-infected leaves. Moreover, due to the induction of many transporters, overexpression of a single gene will not likely lead to complementation of the phenotype.

In conclusion, our findings suggest that bZIP11 functions as a susceptibility factor during $P$. syringae pv. tomato strain DC3000 infection in Arabidopsis. Reduced bZIP11 expression limits $P$. syringae pv. tomato strain DC3000 growth, while increased bZIP11 expression increases growth. These data indicate that bZIP11-regulated metabolic networks may provide nutrients to fuel pathogen reproduction at the local infection site or to regulate host defense responses. Further insight into the role of plant secretory programs in plant disease susceptibility and how they may be coopted by pathogens may offer new strategies to engineer pathogen resistance in crop plants.

\section{MATERIALS AND METHODS}

\section{Bioinformatics.}

Comparison of 76 Arabidopsis seed developmental transcription factors and 74 Arabidopsis transcription factors induced in leaves by $P$. syringae pv. tomato strain DC3000 was completed in Microsoft Excel to identify candidate bZIPI1. Analysis of SWEET and UmamiT promoters was completed using Pscan (Zambelli et al. 2009).

\section{DNA constructs.}

The bZIP11 amiRNA sequence was generated using the Weigelworld online web MicroRNA designer. The amiRNA precursor was produced in pRS300 using published protocols (Schwab et al. 2006). The modified amiRNA precursor (sequence $5^{\prime}$ TGGACAAATAAAAACGGCCCG 3') was inserted into pDONOR221 (Thermo Fisher). The amiRNA sequence binds downstream of the quantitative PCR (qPCR) primers for bZIP11. The estradiol-inducible promoter G1090 and the NOS terminator were amplified and cloned into pDONORp4p1r and pDONORp2rp3. PCR amplification was performed with Phusion High-Fidelity DNA Polymerase (Thermo Fisher), confirmed by sequencing, and integrated into destination vector pB7m34GW-R4R3 by multisite gateway technology (Invitrogen). The final recombinant destination vector was confirmed by restriction profile and mobilized into Agrobacterium tumefaciens $\mathrm{C} 58 \mathrm{C} 1$ by electroporation. The plasmid DNA was isolated from agrobacteria and confirmed by restriction analysis before transformation.

\section{Plant material and transformation.}

Arabidopsis thaliana (Col-0) plants were grown on halfstrength Murashige-Skoog (MS) media (Duchefa, Haarlem, The Netherlands) supplemented with $0.8 \%$ agar, $\mathrm{pH}$ 5.8. Seeds were sterilized by chlorine gas and stratified for 3 to 4 days at $4^{\circ} \mathrm{C}$ in the dark. Ten-day-old seedlings were transferred to soil (50\% compost and 50\% vermiculite) and cultivated under fluorescent light ( $16 \mathrm{~h}$ of light, $120 \mu \mathrm{E} \mathrm{m}^{-2} \mathrm{~s}^{-1}$ ) until flowering. The floral dip transformation method was used to stably transform Arabidopsis plants (Clough and Bent 1998). Transgenic plants were selected on half-strength MS media supplemented with $0.8 \%$ plant agar (Duchefa) and $10 \mu \mathrm{M}$ BASTA (Duchefa) before transfer to soil. T2 selfed progeny were tested for segregation of the selectable marker on solid half-strength MS media. Only seedlings of lines showing a 3:1 segregation were considered for further analysis. Homozygous bzip 11-s1 and bzip11-s2 lines were generated by selfing and used for further experimentation. Seedling growth phenotypes were monitored on half-strength MS medium with $50 \mu \mathrm{M}$ estradiol \pm $30 \mathrm{mM}$ sucrose (16 h of light, $120 \mu \mathrm{E} \mathrm{m}^{-2} \mathrm{~s}^{-1}$ ).

\section{RNA and real-time PCR analyses.}

Plant material was frozen in liquid nitrogen and ground using glass beads in a Micro-Dismembrator. Total RNA was isolated and purified using the RNeasy kit (Qiagen) and quantified by NanoDrop. DNase-treated RNA $(1 \mu \mathrm{g})$ was used for cDNA synthesis by oligo-dT primers and MLV reverse transcription (Promega Corp.) enzyme. The Applied Biosystems with ABIPRISM 7900 was used for gene quantification using SYBR Green and used a standard PCR program for amplification $\left(50^{\circ} \mathrm{C}\right.$ for $2 \mathrm{~min}, 95^{\circ} \mathrm{C}$ for $10 \mathrm{~min}$, and 40 cycles of $95^{\circ} \mathrm{C}$ for 
$15 \mathrm{~s}$ and $60^{\circ} \mathrm{C}$ for $1 \mathrm{~min}$ ). Amplicon dissociation curves were recorded after 40 cycles by heating from 60 to $95^{\circ} \mathrm{C}$ with a ramp speed of $1.9^{\circ} \mathrm{C} \mathrm{min}{ }^{-1}$. Target gene transcript levels were calculated relative to the reference gene At 1 g13320, also known as PP2A (Czechowski et al. 2005). RNA was isolated from $P$. syringae pv. tomato strain DC3000-infected leaves using Sigma-Aldrich's Plant Total RNA isolation kit and protocol. For each sample, $1 \mu \mathrm{g}$ of total RNA was reverse transcribed using the Quantitech Reverse Transcription kit and protocol. qPCR analysis was performed using the Syber Green real-time PCR kit. Primers were designed using the Primer3 software (Rozen and Skaletsky 2000). All primer pairs used are shown in Supplementary Table S3.

\section{Microarray analyses.}

Total RNA was isolated from frozen tissues of 7-day-old seedlings treated with $50 \mu \mathrm{M}$ estradiol using a Sigma-AldrichSpectrum Plant Total RNA Kit and quantified by Nanodrop ND-1000 Spectrophotometer analysis. Three biological replicates were used for microarray analysis. The microarray experiments were performed by ServiceXS using AffymetrixATH1 GeneChips. Quality control of scanned arrays was analyzed using $\mathrm{R}$ language, Bioconductor, and Affymetrix Expression Console Software. Bioconductor was used for robust multiarray average normalization of raw data at gene level to obtain signal intensity values. The limma package was used for comparing treatments and differential expression analysis (Smyth 2005). For functional analysis, g:profiler was used. Raw data can be found in the Gene Expression Omnibus respository under accession number GSE139821.

\section{RNAseq analyses.}

Three replicates of the RNA samples from 7-day-old seedlings treated with $50 \mu \mathrm{M}$ estradiol were used for library preparation (TruSeq RNA Sample Prep Kit v2) and sequenced using Illumina HiSeq2500, single read, $1 \times 50 \mathrm{bp}$. Library preparation and sequencing was performed by Macrogen Europe BV (Amsterdam, The Netherlands). Raw sequencing reads were aligned to the Arabidopsis genome (TAIR10) using TopHat v2.0.13 (Trapnell et al. 2009) with the parameter settings 'bowtie1', 'no-novel-juncs', 'p 6', 'G', 'min-intron-length 40', and 'max-intron-length 2,000'. On average, $97.0 \%$ (94.0 to $98.1 \%$ ) of the RAW reads could be aligned to the genome per biological replicate. This represents an average of 51.1 (40.7 to 73.3) million mapped reads. Aligned reads were summarized over annotated gene models using HTSeq-count v0.6.1 (Anders and Huber 2010) with settings '-stranded no' and '-i gene_id'. From the TAIR10 GTF file, all open reading frames (ORFs) of which the annotation starts with 'CPuORF' were manually removed prior summarization to avoid misalignment of polycistronic mRNAs. Normalized counts were imported to LIMMA (Ritchie et al. 2015) for statistical determination of differential gene expression. Genes for the normalized count that changed more than twofold with an associated $P$ value $<0.05$ (BonferroniHolm test corrected) were considered to be differentially expressed. For functional analysis, g:profiler was used. Raw data can be found online under accession number GSE135593.

\section{Bacterial infection assays.}

For infection assays with $P$. syringae pv. tomato strain DC3000, seed were vernalized for 2 days at $4^{\circ} \mathrm{C}$. Plants were grown for 6.5 weeks in a Percival growth chamber, model I22NLC8, set at a cycle of $10 \mathrm{~h}$ of light $\left(175 \mu \mathrm{E} \mathrm{m}^{-2} \mathrm{~s}^{-1}\right.$ at $\left.22^{\circ} \mathrm{C}\right)$ and $14 \mathrm{~h}$ of darkness and $80 \%$ humidity and watered once a week. For the dex-inducible bZIP11 overexpression line studies, a Percival growth chamber, model CU36L4, was used with the same settings. Plants were fertilized with Peters Excel water-soluble 15-5-15 fertilizer (Scotts-Sierra Horticultural Products Company, Marysville, OH, U.S.A.) at week 2, then every other week until 6.5 weeks of age. Plants were selected such that all Col-0 wild-type, bzip11-s1, and bzip11-s2 plants within each genotype were of similar size, to control for variation in plant size resulting from leaky $b Z I P 11$ expression among individual plants. The Col-0 control and bzip11-s1 individual plants chosen for infection were of comparable size at 6.5 weeks of growth while the bzip11-s2 plants were slightly smaller. Plants were inoculated with a suspension of $P$. syringae pv. tomato DC3000 at $2 \times 10^{6} \mathrm{CFU} / \mathrm{ml}$ in $1 \mathrm{mM} \mathrm{MgCl} 2,5 \mu \mathrm{M}$ estradiol, and 1 or $10 \mu \mathrm{M}$ dexamethasone. The $h r c U$ mutant was inoculated in an identical manner and concentration as wild-type $P$. syringae pv. tomato DC3000 for the growth assays. No drug was used for real-time PCR analysis of infected tissue. Target gene transcript levels were calculated relative to the reference gene At1G49240 (ACTIN 8) for infected leaf tissue. The leaf samples for the qPCR analysis were collected from the same inoculated plants at three time points. A minimum of three individual plants were used for each independent biological replicate. Each time point was obtained by harvesting at least three individual leaves from at least three individual plants and pooling them for a single sample for that specific time point. The mock was infiltration with $1 \mathrm{mM} \mathrm{MgCl}$. Infected plants were placed in a covered flat with $200 \mathrm{ml}$ of standing water and incubated for 3 days for the growth assay. Cork borers $(4.88 \mathrm{~mm}$ in diameter) were used to isolate four leaf discs from each leaf. The leaf discs were ground and suspended in $1 \mathrm{mM} \mathrm{MgCl}_{2}$ for manual $\mathrm{CFU}$ counting. For the qPCR analysis in infected leaves, the inoculation suspension was at a higher concentration of wild-type $P$. syringae pv. tomato DC3000 of $2 \times 10^{8} \mathrm{CFU} / \mathrm{ml}$.

\section{Statistical analysis.}

For the growth assays of wild-type $P$. syringae pv. tomato DC3000 and the $h r c U$ mutant, the two-tailed Student's $t$ test (type II) was used and the $P$ values are given in the corresponding figures.

\section{Oxidative burst assays.}

Three soil-grown, 4-week-old plants were selected for each line. Two leaves per plant were harvested from the youngest fully expanded leaves. Two leaf discs ( $5 \mathrm{~mm}$ in diameter) were taken from each harvested leaf $(n=12)$ and incubated in water in a 96-well plate (one leaf disc per well) for $24 \mathrm{~h}$. To measure ROS, leaf discs were treated with flg22 $(100 \mathrm{nM})$ in a solution of horseradish peroxidase at $10 \mu \mathrm{g} / \mathrm{ml}$ and $100 \mu \mathrm{M}$ Luminol (Sigma-Aldrich), then immediately subjected to luminescence measurement with a 1420 Multilabel Counter (Perkin Elmer). Relative luminescence units are reported. Assays were repeated three times independently with similar results.

\section{ACKNOWLEDGMENTS}

We thank A. Jones (University of Cambridge), L.-Q. Chen (University of Illinois at Urbana-Champaign), and D. Sosso for many useful discussions.

\section{AUTHOR-RECOMMENDED INTERNET RESOURCES}

\footnotetext{
Affymetrix Expression Console Software: https://www.thermofisher.com/ us/en/home/life-science/microarray-analysis.html

Bioconductor: https://www.bioconductor.org

Gene Expression Omnibus: https://www.ncbi.nlm.nih.gov/geo g:profiler: https://biit.cs.ut.ee/gprofiler/gost

MicroRNA designer: http://wmd3.weigelworld.org/cgi-bin/webapp.cgi ServiceXS: https://www.genomescan.nl
} 


\section{LITERATURE CITED}

Anders, S., and Huber, W. 2010. Differential expression analysis for sequence count data. Genome Biol. 11:R106.

Anderson, J. C., Bartels, S., González Besteiro, M. A., Shahollari, B., Ulm, R., and Peck, S. C. 2011. Arabidopsis MAP kinase phosphatase 1 (AtMKP1) negatively regulates MPK6-mediated PAMP responses and resistance against bacteria. Plant J. 67:258-268.

Bartels, S., Anderson, J. C., González Besteiro, M. A., Carreri, A., Hirt, H., Buchala, A., Métraux, J. P., Peck, S. C., and Ulm, R. 2009. MAP kinase phosphatase1 and protein tyrosine phosphatase1 are repressors of salicylic acid synthesis and SNC1-mediated responses in Arabidopsis. Plant Cell 21:2884-2897.

Besnard, J., Zhao, C., Avice, J.-C., Vitha, S., Hyodo, A., Pilot, G., and Okumoto, S. 2018. Arabidopsis UMAMIT24 and 25 are amino acid exporters involved in seed loading. J. Exp. Bot. 69:5221-5232.

Bezrutczyk, M., Yang, J., Eom, J.-S., Prior, M., Sosso, D., Hartwig, T., Szurek, B., Oliva, R., Vera-Cruz, C., White, F. F., Yang, B., and Frommer, W. B. 2018. Sugar flux and signaling in plant-microbe interactions. Plant J. 93:675-685.

Buell, C. R., Joardar, V., Lindeberg, M., Selengut, J., Paulsen, I. T., Gwinn, M. L., Dodson, R. J., Deboy, R. T., Durkin, A. S., Kolonay, J. F., Madupu, R., Daugherty, S., Brinkac, L., Beanan, M. J., Haft, D. H., Nelson, W. C., Davidsen, T., Zafar, N., Zhou, L., Liu, J., Yuan, Q., Khouri, H., Fedorova, N., Tran, B., Russell, D., Berry, K., Utterback, T., Van Aken, S. E., Feldblyum, T. V., D’Ascenzo, M., Deng, W. L., Ramos, A. R., Alfano, J. R., Cartinhour, S., Chatterjee, A. K., Delaney, T. P., Lazarowitz, S. G., Martin, G. B., Schneider, D. J., Tang, X., Bender, C. L., White, O., Fraser, C. M., and Collmer, A. 2003. The complete genome sequence of the Arabidopsis and tomato pathogen Pseudomonas syringae pv. tomato DC3000. Proc. Natl. Acad. Sci. U.S.A. 100:10181-10186.

Cernadas, R. A., Doyle, E. L., Niño-Liu, D. O., Wilkins, K. E., Bancroft, T., Wang, L., Schmidt, C. L., Caldo, R., Yang, B., White, F. F., Nettleton, D., Wise, R. P., and Bogdanove, A. J. 2014. Code-assisted discovery of TAL effector targets in bacterial leaf streak of rice reveals contrast with bacterial blight and a novel susceptibility gene. PLoS Pathog. 10: e1003972.

Chen, L. Q., Hou, B. H., Lalonde, S., Takanaga, H., Hartung, M. L., Qu, X. Q., Guo, W. J., Kim, J. G., Underwood, W., Chaudhuri, B., Chermak, D., Antony, G., White, F. F., Somerville, S. C., Mudgett, M. B., and Frommer, W. B. 2010. Sugar transporters for intercellular exchange and nutrition of pathogens. Nature 468:527-532.

Chen, L. Q., Lin, I. W., Qu, X. Q., Sosso, D., McFarlane, H. E., Londoño, A., Samuels, A. L., and Frommer, W. B. 2015. A cascade of sequentially expressed sucrose transporters in the seed coat and endosperm provides nutrition for the Arabidopsis embryo. Plant Cell 27:607-619.

Chong, J., Piron, M. C., Meyer, S., Merdinoglu, D., Bertsch, C., and Mestre, P. 2014. The SWEET family of sugar transporters in grapevine: VvSWEET4 is involved in the interaction with Botrytis cinerea. J. Exp. Bot. 65:6589-6601.

Clough, S. J., and Bent, A. F. 1998. Floral dip: A simplified method for Agrobacterium-mediated transformation of Arabidopsis thaliana. Plant J. 16:735-743.

Cohn, M., Bart, R. S., Shybut, M., Dahlbeck, D., Gomez, M., Morbitzer, R., Hou, B. H., Frommer, W. B., Lahaye, T., and Staskawicz, B. J. 2014. Xanthomonas axonopodis virulence is promoted by a transcription activator-like effector-mediated induction of a SWEET sugar transporter in cassava. Mol. Plant-Microbe Interact. 27:1186-1198.

Cox, K. L., Meng, F., Wilkins, K. E., Li, F., Wang, P., Booher, N. J., Carpenter, S. C. D., Chen, L. Q., Zheng, H., Gao, X., Zheng, Y., Fei, Z., Yu, J. Z., Isakeit, T., Wheeler, T., Frommer, W. B., He, P., Bogdanove, A. J., and Shan, L. 2017. TAL effector driven induction of a SWEET gene confers susceptibility to bacterial blight of cotton. Nat. Commun. 8:15588.

Cumbie, J. S., Kimbrel, J. A., Di, Y., Schafer, D. W., Wilhelm, L. J., Fox, S. E., Sullivan, C. M., Curzon, A. D., Carrington, J. C., Mockler, T. C., and Chang, J. H. 2011. GENE-counter: A computational pipeline for the analysis of RNA-Seq data for gene expression differences. PLoS One 6:e25279.

Czechowski, T., Stitt, M., Altmann, T., Udvardi, M. K., and Scheible, W.-R. 2005. Genome-wide identification and testing of superior reference genes for transcript normalization in Arabidopsis. Plant Physiol. 139:5-17.

Dou, D., and Zhou, J.-M. 2012. Phytopathogen effectors subverting host immunity: Different foes, similar battleground. Cell Host Microbe 12: 484-495.

Eom, J. S., Luo, D., Atienza-Grande, G., Yang, J., Ji, C., Thi Luu, V., Huguet-Tapia, J. C., Char, S. N., Liu, B., Nguyen, H., Schmidt, S. M., Szurek, B., Vera Cruz, C., White, F. F., Oliva, R., Yang, B., and Frommer, W. B. 2019. Diagnostic kit for rice blight resistance. Nat. Biotechnol. 37: 1372-1379.
Hahn, M., Viaud, M., and Kan, J. V. 2014. The Genome of Botrytis cinerea, a ubiquitous broad host range necrotroph. Pages 19-44 in: Genomics of Plant-Associated Fungi and Oomycetes: Dicot Pathogens. R. A. Dean, A. Lichens-Park, and C. Kole, eds. Springer, Berlin, Heidelberg, Germany. doi:10.1007/978-3-662-44056-8_2

Hanson, J., Hanssen, M., Wiese, A., Hendriks, M. M. W. B., and Smeekens, S. 2008. The sucrose regulated transcription factor bZIP11 affects amino acid metabolism by regulating the expression of ASPARAGINE SYNTHETASE1 and PROLINE DEHYDROGENASE2. Plant J. 53: 935-949.

Herbers, K., Meuwly, P., Frommer, W. B., Metraux, J. P., and Sonnewald, U. 1996. Systemic acquired resistance mediated by the ectopic expression of invertase: Possible hexose sensing in the secretory pathway. Plant Cell 8:793-803.

Huai, B., Yang, Q., Wei, X., Pan, Q., Kang, Z., and Liu, J. 2020. TaSTP13 contributes to wheat susceptibility to stripe rust possibly by increasing cytoplasmic hexose concentration. BMC Plant Biol. 20:49.

Huang, H. C., He, S. Y., Bauer, D. W., and Collmer, A. 1992. The Pseudomonas syringae pv. syringae $61 \mathrm{hrpH}$ product, an envelope protein required for elicitation of the hypersensitive response in plants. J. Bacteriol. 174:6878-6885.

Jones, J. D. G., and Dangl, J. L. 2006. The plant immune system. Nature 444:323-329.

Kemmerling, B., Schwedt, A., Rodriguez, P., Mazzotta, S., Frank, M., Qamar, S. A., Mengiste, T., Betsuyaku, S., Parker, J. E., Müssig, C., Thomma, B. P., Albrecht, C., de Vries, S. C., Hirt, H., and Nürnberger, T. 2007. The BRI1-associated kinase 1, BAK1, has a brassinolideindependent role in plant cell-death control. Curr. Biol. 17:1116-1122.

Kocal, N., Sonnewald, U., and Sonnewald, S. 2008. Cell wall-bound invertase limits sucrose export and is involved in symptom development and inhibition of photosynthesis during compatible interaction between tomato and Xanthomonas campestris pv vesicatoria. Plant Physiol. 148:1523-1536.

Ladwig, F., Stahl, M., Ludewig, U., Hirner, A. A., Hammes, U. Z., Stadler, R., Harter, K., and Koch, W. 2012. Siliques are Red1 from Arabidopsis acts as a bidirectional amino acid transporter that is crucial for the amino acid homeostasis of siliques. Plant Physiol. 158:1643-1655.

Le, B. H., Cheng, C., Bui, A. Q., Wagmaister, J. A., Henry, K. F., Pelletier, J., Kwong, L., Belmonte, M., Kirkbride, R., Horvath, S., Drews, G. N., Fischer, R. L., Okamuro, J. K., Harada, J. J., and Goldberg, R. B. 2010. Global analysis of gene activity during Arabidopsis seed development and identification of seed-specific transcription factors. Proc. Natl. Acad. Sci. U.S.A. 107:8063-8070.

Lim, C. W., Baek, W., Lim, S., Han, S. W., and Lee, S. C. 2015. Expression and functional roles of the pepper pathogen-induced bZIP transcription factor CabZIP2 in enhanced disease resistance to bacterial pathogen infection. Mol. Plant-Microbe Interact. 28:825-833.

Liu, X., Zhang, Y., Yang, C., Tian, Z., and Li, J. 2016. AtSWEET4, a hexose facilitator, mediates sugar transport to axial sinks and affects plant development. Sci. Rep. 6:24563.

Ma, J., Hanssen, M., Lundgren, K., Hernández, L., Delatte, T., Ehlert, A., Liu, C. M., Schluepmann, H., Dröge-Laser, W., Moritz, T., Smeekens, S., and Hanson, J. 2011. The sucrose-regulated Arabidopsis transcription factor bZIP11 reprograms metabolism and regulates trehalose metabolism. New Phytol. 191:733-745.

Mathan, J., Singh, A., and Ranjan, A. Sucrose transport in response to drought and salt stress involves ABA-mediated induction of OsSWEET13 and OsSWEET15 in rice. Physiol. Plant. In press. doi.org/10.1111/ppl.13210

Moore, J. W., Herrera-Foessel, S., Lan, C., Schnippenkoetter, W., Ayliffe, M., Huerta-Espino, J., Lillemo, M., Viccars, L., Milne, R., Periyannan, S., Kong, X., Spielmeyer, W., Talbot, M., Bariana, H., Patrick, J. W., Dodds, P., Singh, R., and Lagudah, E. 2015. A recently evolved hexose transporter variant confers resistance to multiple pathogens in wheat. Nat. Genet. 47:1494-1498.

Mudgett, M. B., and Staskawicz, B. J. 1999. Characterization of the Pseudomonas syringae pv. tomato AvrRpt2 protein: Demonstration of secretion and processing during bacterial pathogenesis. Mol. Microbiol. 32:927-41.

Müller, B., Fastner, A., Karmann, J., Mansch, V., Hoffmann, T., Schwab, W., Suter-Grotemeyer, M., Rentsch, D., Truernit, E., Ladwig, F., Bleckmann, A., Dresselhaus, T., and Hammes, U. Z. 2015. Amino acid export in developing Arabidopsis seeds depends on UmamiT facilitators. Curr. Biol. 25:3126-3131.

Oliva, R., Ji, C., Atienza-Grande, G., Huguet-Tapia, J. C., Perez-Quintero, A., Li, T., Eom, J. S., Li, C., Nguyen, H., Liu, B., Auguy, F., Sciallano, C., Luu, V. T., Dossa, G. S., Cunnac, S., Schmidt, S. M., Slamet-Loedin, I. H., Vera Cruz, C., Szurek, B., Frommer, W. B., White, F. F., and Yang, 
B. 2019. Broad-spectrum resistance to bacterial blight in rice using genome editing. Nat. Biotechnol. 37:1344-1350.

Ritchie, M. E., Phipson, B., Wu, D., Hu, Y., Law, C. W., Shi, W., and Smyth, G. K. 2015. limma Powers differential expression analyses for RNAsequencing and microarray studies. Nucleic Acids Res. 43:e47.

Rozen, S., and Skaletsky, H. 2000. Primer3 on the WWW for general users and for biologist programmers. Pages 365-386 in: Bioinformatics Methods and Protocols. Methods in Molecular Biology, Vol. 132. S. Misener and S. A. Krawetz, eds. Humana Press, Totowa, NJ, U.S.A. doi: 10.1385/1-59259-192-2:365

Schwab, R., Ossowski, S., Riester, M., Warthmann, N., and Weigel, D. 2006. Highly specific gene silencing by artificial microRNAs in Arabidopsis. Plant Cell 18:1121-1133.

Schwelm, A., Fogelqvist, J., Knaust, A., Jülke, S., Lilja, T., Bonilla-Rosso, G., Karlsson, M., Shevchenko, A., Dhandapani, V., Choi, S. R., Kim, H. G., Park, J. Y., Lim, Y. P., Ludwig-Müller, J., and Dixelius, C. 2015. The Plasmodiophora brassicae genome reveals insights in its life cycle and ancestry of chitin synthases. Sci. Rep. 5:11153.

Siemens, J., Keller, I., Sarx, J., Kunz, S., Schuller, A., Nagel, W., Schmülling, T., Parniske, M., and Ludwig-Müller, J. 2006. Transcriptome analysis of Arabidopsis clubroots indicate a key role for cytokinins in disease development. Mol. Plant-Microbe Interact. 19:480-494.

Smyth, G. K. 2005. limma: Linear models for microarray data. Pages 397-420 in: Bioinformatics and Computational Biology Solutions Using $\mathrm{R}$ and Bioconductor. Statistics for Biology and Health. R. Gentleman, V. J. Carey, W. Huber, R. A. Irizarry, and S. Dudoit, eds. Springer, New York, NY, U.S.A. doi:10.1007/0-387-29362-0_23

Sonnewald, S., Priller, J. P., Schuster, J., Glickmann, E., Hajirezaei, M. R., Siebig, S., Mudgett, M. B., and Sonnewald, U. 2012. Regulation of cell wall-bound invertase in pepper leaves by Xanthomonas campestris pv. vesicatoria type three effectors. PLoS One 7:e51763.
Thalor, S. K., Berberich, T., Lee, S. S., Yang, S. H., Zhu, X., Imai, R., Takahashi, Y., and Kusano, T. 2012. Deregulation of sucrose-controlled translation of a bZIP-type transcription factor results in sucrose accumulation in leaves. PLoS One 7:e33111.

Thilmony, R., Underwood, W., and He, S. Y. 2006. Genome-wide transcriptional analysis of the Arabidopsis thaliana interaction with the plant pathogen Pseudomonas syringae pv. tomato DC3000 and the human pathogen Escherichia coli O157:H7. Plant J. 46:34-53.

Trapnell, C., Pachter, L., and Salzberg, S. L. 2009. TopHat: Discovering splice junctions with RNA-Seq. Bioinformatics 25:1105-1111.

Trujillo, M., Ichimura, K., Casais, C., and Shirasu, K. 2008. Negative regulation of PAMP-triggered immunity by an E3 ubiquitin ligase triplet in Arabidopsis. Curr. Biol. 18:1396-1401.

Truman, W., de Zabala, M. T., and Grant, M. 2006. Type III effectors orchestrate a complex interplay between transcriptional networks to modify basal defence responses during pathogenesis and resistance. Plant J. 46:14-33.

van Schie, C. C. N., and Takken, F. L. W. 2014. Susceptibility genes 101: How to be a good host. Annu. Rev. Phytopathol. 52:551-581.

Yamada, K., Saijo, Y., Nakagami, H., and Takano, Y. 2016. Regulation of sugar transporter activity for antibacterial defense in Arabidopsis. Science 354:1427-1430.

Zambelli, F., Pesole, G., and Pavesi, G. 2009. Pscan: Finding overrepresented transcription factor binding site motifs in sequences from co-regulated or co-expressed genes. Nucleic Acids Res. 37: W247-W252.

Zeng, L.-R., Qu, S., Bordeos, A., Yang, C., Baraoidan, M., Yan, H., Xie, Q., Nahm, B. H., Leung, H., and Wang, G.-L. 2004. Spotted leaf11, a negative regulator of plant cell death and defense, encodes a U-box/armadillo repeat protein endowed with E3 ubiquitin ligase activity. Plant Cell 16:2795-2808. 